\title{
Different patterns of cardiac sympathetic denervation in tremor-type compared to akinetic-rigid-type Parkinson's disease: Molecular imaging with ${ }^{123}$ I-MIBG
}

\author{
A. CHIARAVALLOTI ${ }^{1}$, A. STEFANI ${ }^{2,3}$, M. TAVOLOZZA ${ }^{1}$, M. PIERANTOZZI ${ }^{2,3}$, D. DI BIAGIO ${ }^{1}$, E. OLIVOLA ${ }^{2,3}$, \\ B. DI PIETRO ${ }^{1}$, M. STAMPANONI ${ }^{2,3}$, R. DANIELI ${ }^{1}$, G. SIMONETTI ${ }^{1}$, P. STANZIONE ${ }^{2,3}$ and O. SCHILLACI ${ }^{1,4}$ \\ Departments of ${ }^{1}$ Biopathology and Diagnostic Imaging and ${ }^{2}$ Neurological Sciences, University Tor Vergata, Rome; \\ ${ }^{3}$ IRCCS Fondazione S. Lucia, Rome; ${ }^{4}$ IRCCS Neuromed, Pozzilli, Italy
}

Received February 28, 2012; Accepted August 21, 2012

DOI: $10.3892 / \mathrm{mmr} .2012 .1104$

\begin{abstract}
The aim of this study was to evaluate the correlation between the clinical motor phenotypes of Parkinson's disease (PD) and ${ }^{123} \mathrm{I}-\mathrm{MIBG}$ myocardial uptake. In total, 53 patients with PD [31 males and 22 females, mean age 62 \pm 10 years; 19 Hoehn \& Yahr (H\&Y) stage 1, 9 stage 1.5, 15 stage 2 and 10 at stage 3] were examined and subdivided into different clinical forms on the basis of dominance of resting tremor $(n=19$, TDT) and bradykinesia plus rigidity $(n=34$, ART). This status was correlated with the semi-quantitative analysis of ${ }^{123}$ I-MIBG myocardial uptake. An age-matched control group of 18 patients was recruited ( 8 males and 10 females, mean age $62.4 \pm 16.3$ years). ${ }^{123}$ I-MIBG myocardial uptake significantly correlated with disease duration in early $\left(\mathrm{r}^{2}=0.1894 ; \mathrm{P}=0.0028\right)$ and delayed images $\left(\mathrm{r}^{2}=0.1795 ; \mathrm{P}=0.0037\right)$ in $\mathrm{PD}$ patients, while no correlation was found when considering age at examination, UPDRS III motor examination section score and H\&Y score. PD patients showed a reduced ${ }^{123} \mathrm{I}-\mathrm{MIBG}$ myocardial uptake compared to the control group in early $(\mathrm{P}=0.0026)$ and delayed images $(\mathrm{P}=0.0040)$, and ${ }^{123} \mathrm{I}-\mathrm{MIBG}$ myocardial uptake was significantly lower in delayed images in TDT patients compared with ART patients $(\mathrm{P}=0.0167)$. A decrease was detected in the heart-to-mediastinum $(\mathrm{H} / \mathrm{M})$ ratio in delayed images compared to that of the early images in TDT patients $(\mathrm{P}=0.0040)$ and in the whole $\mathrm{PD}$ population $(\mathrm{P}=0.0012)$, while no differences were found in ART patients $(\mathrm{P}=0.1043)$. The results of the present study revealed that the cardiac sympathetic system is more severely impaired in TDT than in ART patients and ${ }^{123} \mathrm{I}-$ MIBG molecular imaging has the potential help in improving therapeutic planning in these patients.
\end{abstract}

Correspondence to: Professor Orazio Schillaci, Department of Biopathology and Diagnostic Imaging, University Tor Vergata, Viale Oxford 8100173 Rome, Italy

E-mail: orazio.schillaci@uniroma2.it

Key words: ${ }^{123}$ I-MIBG, Parkinson's disease, tremor-type, akinetic rigid type, sympathetic system

\section{Introduction}

${ }^{123}$ I-metaiodobenzylguanidine $\left({ }^{123} \mathrm{I}-\mathrm{MIBG}\right)$, a physiological analogue of noradrenaline, is actively transported into noradrenaline granules at sympathetic nerve terminals by a noradrenaline transporter and allows the quantification of cardiac sympathetic innervation in vivo (1). In Parkinson's disease (PD), neuronal damage in the areas associated with motor functions, particularly the superordinate centers of the somatomotor, visceromotor and limbic systems, has been reported (2). In particular, structures such as the dorsal motor nucleus of the vagal nerve and intermediate reticular zone are involved in the early stages, while the substantia nigra and other nuclear grays of the midbrain and forebrain become the focus of initially slight, and then severe, pathological changes in the late stages (2). These findings are consistent with cardiac sympathetic denervation shown by means of myocardial ${ }^{123}$ I-MIBG scintigraphy, indicating an impairment of the nigrostriatal dopaminergic and myocardial sympathetic systems in the majority of the patients with PD, even in the earliest clinical stage of the disease (3).

In total, $50 \%$ of PD patients complain of slowness of movement at presentation (4). The symptoms of PD involve the initiation and execution of movements, particularly sequential and volitional actions (4). Approximately $40 \%$ of patients are likely to complain of tremulousness of the hand at rest, which improves with action and $80 \%$ of patients have an asymmetrical 3-5 Hz rest tremor evident on examination (5). The two subtypes of PD have different courses, which are concordant with the differences in brain biochemical abnormalities (5), and it has been reported that PD progresses more rapidly in akinetic rigid-type (ART) patients than in tremor dominant-type (TDT) patients (6). Previous studies suggested that ${ }^{123} \mathrm{I}-\mathrm{MIBG}$ cardiac uptake is increased in PD patients with bradykinesia as compared with tremor patients (7). Additionally, Spiegel et al found that at all Hoen \& Yahr (H\&Y) stages, myocardial MIBG uptake was significantly higher in TDT patients than in ART patients (8).

In this study, ${ }^{123}$ I-MIBG myocardial uptake was compared in patients with different subtypes of PD in order to assess the correlation between the clinical phenotype and sympathetic 
denervation. Moreover, the results concerning the PD population were evaluated in comparison with those of a control group (CG).

\section{Materials and methods}

Patients. The study involved 53 patients with PD [31 males and 22 females, mean age $62 \pm 10$ years; 19 H\&Y stage 1 , 9 stage $1.5,15$ stage 2 and 10 at stage 3]. A clinical examination was conducted by an experienced neurologist (P.S.) and PD was diagnosed using United Kingdom Parkinson's Disease Society Brain Bank (UKPDSBB) criteria (9). The motor part of the UKPDSBB criteria was used to assess the severity of disease (10). On the basis of the predominant motor features in UPDRS III motor examination section score, patients were subtyped into one of two clinical groups, ART and TDT, following the methods described in previous studies (11-14). The Mini Mental State Examination (MMSE) did not reveal any cognitive deficit. Nineteen patients had TDT and 34 had ART. A 'tremor score' and a 'non-tremor score' were calculated for each patient $(11,15)$. The data concerning the clinical subtypes of patients with PD are shown in Table I. The tremor score was derived from the sum of UPDRS items 20 (tremor at rest) and 21 (action or postural tremor of hands) divided by 7 (the number of the single subitems included). The non-tremor score was derived from the sum of UPDRS items 18 (speech), 19 (facial expression), 22 (rigidity), 27 (arising from the chair), 28 (posture), 29 (gait), 30 (postural stability) and 31 (body bradykinesia and hypokinesia) divided by 12 (the number of the single subitems included). The patient was classified as tremor-dominant type when the tremor score was at least twice the non-tremor score. An age-matched control group (CG) of 18 patients was recruited (8 males and 10 females, mean age $62.4 \pm 16.3$ years). The $C G$ patients underwent a ${ }^{123}$ I-MIBG scintigraphy for a suspected adrenal mass, which was then revealed to be negative on image evaluation. Prior to their inclusion in our study, the subjects were evaluated for the absence of clinical signs of PD by an experienced neurologist (A.S.). Exclusion criteria for the PD and CG patients included diabetes, a history of neuropathy, previous relevant cardiac disease or any other medical condition that potentially affected the autonomic nervous system or the myocardial ${ }^{123} \mathrm{I}-$ MIBG uptake. None of the subjects were undergoing treatment with drugs that have been previously reported to affect ${ }^{123} \mathrm{I}$ MIBG uptake at the sympathetic terminals $(15,16)$. Informed consent was obtained in all cases from the patients themselves or from their families, in accordance with the Declaration of Helsinki. This study was approved by the ethics committee of the University of Tor Vergata.

Data acquisition. Each PD patient was intravenously injected in $60 \mathrm{sec}$ with $111 \mathrm{MBq}$ of ${ }^{123} \mathrm{I}-\mathrm{MIBG}$ (Amersham, Eindhoven, The Netherlands) and 185-200 MBq was injected in CG patients in $5 \mathrm{~min}$. The radio-labeled compound was administered at the same time of the day under the same experimental conditions in each patient.

Data were collected by means of a dual head gammacamera (Millennium VG; General Electric Medical Systems, Milwaukee, WI, USA) equipped with low-energy highresolution parallel-hole collimators with static planar images in a $128 \times 128$ matrix. Images were captured 30 min (early images) and $4 \mathrm{~h}$ (delayed images) after the injection of the radio-labeled compound (Fig. 1). A region of interest (ROI) was manually drawn over the myocardium, including the left ventricular cavity. ROIs were then set over the upper mediastinum and a heart-to-mediastinum (H/M) count-ratio was calculated, which was defined as the average counts/pixel in the myocardium, divided by that of the upper mediastinum (1).

Statistical analysis. The means and standard deviation were calculated for age, UPDRS III score, H\&Y grade, disease duration and the results of the semi-quantitative analysis for the early and delayed H/M count-ratio. To detect differences in the cardiac sympathetic innervation between the subgroups (semi-quantitative analysis, non-parametric data) a Mann-Whitney U test was used. Differences in early and delayed $\mathrm{H} / \mathrm{M}$ in the PD subgroups (ART and TDT) and CG patients were analyzed by means of a Wilcoxon matched-pairs test. The Spearman's correlation was used to determine the relationship among UPDRS motor scores, H\&Y stage, age at examination and early and delayed $\mathrm{H} / \mathrm{M}$ count-ratio. Multiple regression analysis was applied to observe the effect of disease duration. The Chi-square test was used to assess differences in the H\&Y stage and disease phenotype. An unpaired t-test was used to assess differences between age, disease duration, UPDRS and disease phenotype. A hypothesis was considered valid when $\mathrm{P} \leq 0.05$.

\section{Results}

Patient characteristics and analysis of early and delayed $H / M$ count-ratio. Differences in age, gender, disease duration and H\&Y stage between ART and TDT patients were not significant. Patient characteristics are shown in Table I.

The results of the semi-quantitative analysis of the early and delayed H/M count-ratio are shown in Table II. A reduced ${ }^{123}$ I-MIBG myocardial uptake was detected in PD patients as compared to $\mathrm{CG}$ patients in early $(\mathrm{P}=0.0026)$ and delayed images $(\mathrm{P}=0.0040)$. The $\mathrm{H} / \mathrm{M}$ ratio was lower in ART patients as compared to $\mathrm{CG}$ patients only in early images $(\mathrm{P}=0.0017)$, while no statistically significant differences were observed in delayed acquisitions $(\mathrm{P}=0.0691)$. A lower $\mathrm{H} / \mathrm{M}$ ratio was detected in TDT patients as compared to the CG ones in both the early and delayed images $(\mathrm{P}=0.0016 ; \mathrm{P}=0.0002)$. A significant decrease in the $\mathrm{H} / \mathrm{M}$ ratio was found between delayed images as compared to that of the early images in TDT patients $(\mathrm{P}=0.0040), \mathrm{CG}$ patients $(\mathrm{P}=0.0175)$ and in the entire $\mathrm{PD}$ population $(\mathrm{P}=0.0012)$, while no statistically significant differences were found when comparing early and delayed $\mathrm{H} / \mathrm{M}$ ratio in ART patients $(\mathrm{P}=0.1043)$. No statistically significant differences were detected when comparing early $\mathrm{H} / \mathrm{M}$ count ratios in TDT and ART patients $(\mathrm{P}=0.1727)$, while ${ }^{123} \mathrm{I}-\mathrm{MIBG}$ myocardial uptake was significantly lower in delayed images in TDT ( $\mathrm{P}=0.0167)$ compared to ART patients (Fig. 2).

Effects on UPDRS score. The UPDRS score was higher in ART patients as compared with TDT patients $(\mathrm{P}=0.0192)$. The early and delayed $\mathrm{H} / \mathrm{M}$ ratio of $\mathrm{PD}$ patients was not associated with UPDRS ( $\mathrm{r}=-0.1236, \mathrm{P}=0.4297$; $\mathrm{r}=-0.0057$, $\mathrm{P}=0.9708)$. Early and delayed ${ }^{123} \mathrm{I}-\mathrm{MIBG}$ myocardial 
Table I. General overview of the PD population examined.

\begin{tabular}{lccc}
\hline & Population $(\mathrm{n}=53)$ & TDT $(\mathrm{n}=19)$ & ART $(\mathrm{n}=34)$ \\
\hline Gender & & 10 & 21 \\
Males & 31 & 9 & 13 \\
Females & 22 & $66.1 \pm 68.2$ & $41.4 \pm 54.7$ \\
Disease duration (months \pm SD) & $50.7 \pm 60.6$ & $18.2 \pm 6.6$ & $26.3 \pm 12.3$ \\
UPDRS III (mean \pm SD) & $23.3 \pm 11.1$ & & 7 \\
Hoehn \& Yahr score & & & 5 \\
1 & 19 & 4 & 9 \\
1.5 & 9 & 6 & 8 \\
2 & 15 & 2 & 9
\end{tabular}

PD, Parkinson's disease; TDT, tremor-dominant type; ART, akinetic-rigid type; UPDRS III, UPDRS motor examination section score III.

Table II. Early and delayed H/M ratio of the ${ }^{123}$ I-MIBG myocardial uptake in clinical PD subtypes and the CG patients.

\begin{tabular}{|c|c|c|c|c|c|c|}
\hline & CG & PD & TDT & ART & Comparison & P-value \\
\hline Early H/M & $1.97 \pm 0.28$ & $1.68 \pm 0.36$ & $1.59 \pm 0.34$ & $1.72 \pm 0.36$ & $\begin{array}{l}\text { PD vs. CG } \\
\text { TDT vs. CG } \\
\text { ART vs. CG } \\
\text { TDT vs. ART }\end{array}$ & $\begin{array}{l}0.0026 \\
0.0016 \\
0.0017 \\
0.1727\end{array}$ \\
\hline Delayed H/M & $1.88 \pm 0.27$ & $1.58 \pm 0.36$ & $1.43 \pm 0.38$ & $1.66 \pm 0.33$ & $\begin{array}{l}\text { PD vs. CG } \\
\text { TDT vs. CG } \\
\text { ART vs. CG } \\
\text { TDT vs. ART }\end{array}$ & $\begin{array}{l}0.0040 \\
0.0002 \\
0.0691 \\
0.0167\end{array}$ \\
\hline
\end{tabular}

CG, control group; PD, Parkinson's disease; TDT, tremor-dominant type; ART, akinetic-rigid type; H/M, heart-to-mediastinum; ${ }^{123} \mathrm{I}-\mathrm{MIBG}$, ${ }^{123}$ I-metaiodobenzylguanidine. Values are expressed as the means \pm SD.

A

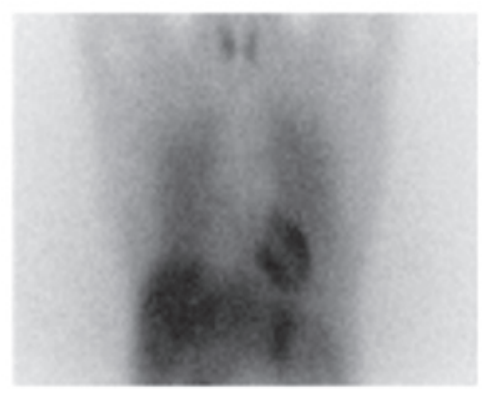

B

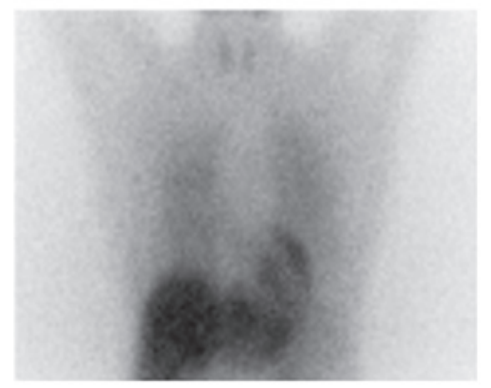

C

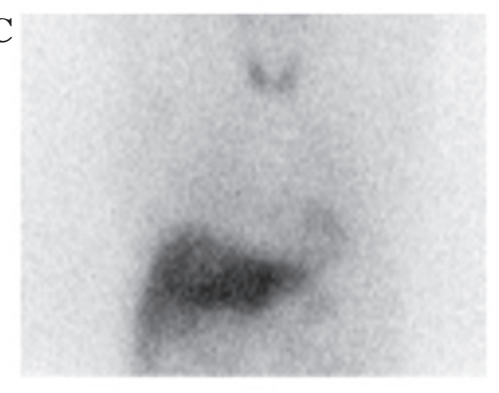

D

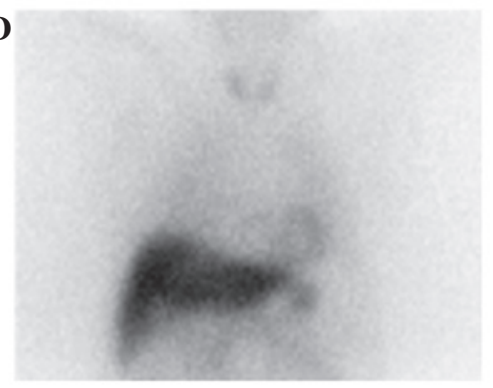

E

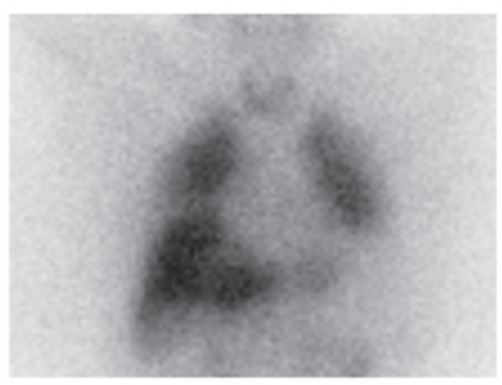

F

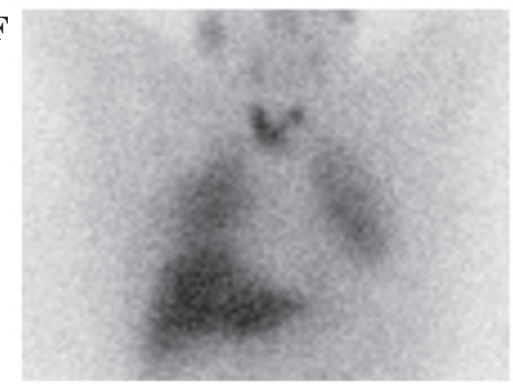

Figure 1. Sample images of ${ }^{123} \mathrm{I}-\mathrm{MIBG}$ myocardial uptake in a CG patient: (A) early, (B) delayed; an ART patient: (C) early, (D) delayed; and a TDT patient: (E) early, (F) delayed. ${ }^{123}$ I-MIBG, ${ }^{123}$ I-metaiodobenzylguanidine; CG, control group; TDT, tremor-dominant type; ART, akinetic-rigid type. 
A

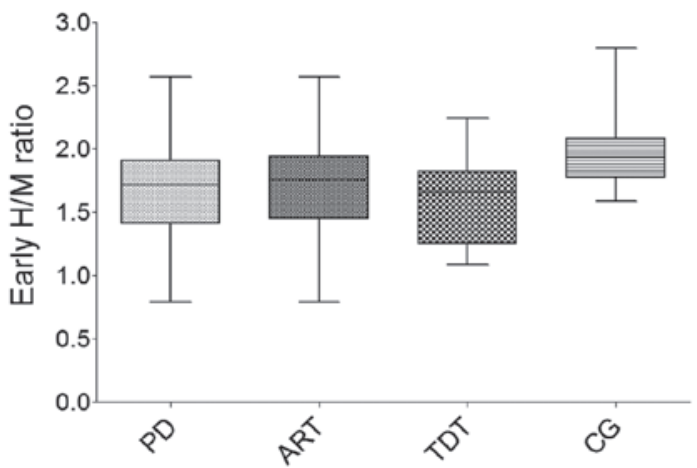

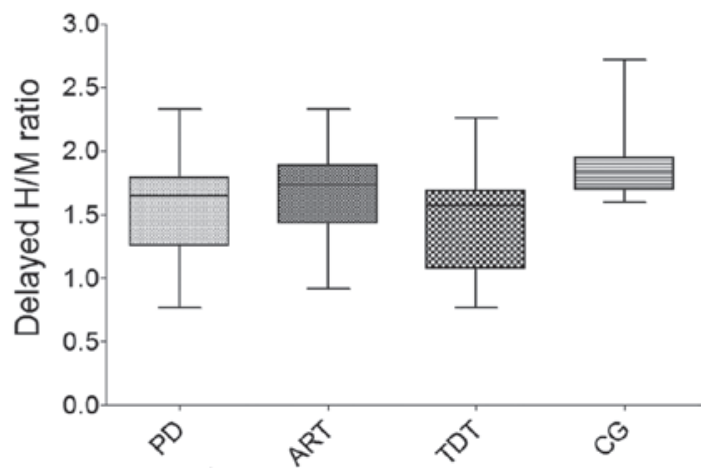

Figure 2. Examination of ${ }^{123} \mathrm{I}-\mathrm{MIBG}$ myocardial uptake in the studied population. (A and B) The early and delayed H/M ratios were lower in the PD population as compared to the CG population. (B) ${ }^{123} \mathrm{I}-\mathrm{MIBG}$ myocardial uptake was significantly lower in delayed images in TDT patients when compared to that in ART patients. ${ }^{123} \mathrm{I}-\mathrm{MIBG},{ }^{123} \mathrm{I}$-metaiodobenzylguanidine; H/M, heart-to-mediastinum; PD, Parkinson's disease; $\mathrm{CG}$, control group; TDT, tremor-dominant type; ART, akinetic-rigid type.

uptake was not correlated with UPDRS in ART ( $\mathrm{r}=-0.3132$, $\mathrm{P}=0.1117 ; \mathrm{r}=-0.2128, \mathrm{P}=0.2865)$ and $\mathrm{TDT}$ patients $(r=0.07985, P=0.7688 ; r=0.04546, P=0.8672)$. The $H / M$ ratio was not associated with $\mathrm{H} \& \mathrm{Y}$ stage in $\mathrm{PD}$ patients in early $(r=-0.2411, P=0.0821)$ or delayed $(r=-0.1969, P=0.1577)$ scans. ${ }^{123} \mathrm{I}-\mathrm{MIBG}$ myocardial uptake was also not correlated with the H\&Y stage in the early $(r=-0.1909, P=0.2794)$ and delayed images $(\mathrm{r}=-0.1491, \mathrm{P}=0.4002)$ in ART patients. No correlation was found between $\mathrm{H} \& \mathrm{Y}$ stage and early $(\mathrm{r}=-0.4047, \mathrm{P}=0.0856)$ and delayed $(\mathrm{r}=-0.4352, \mathrm{P}=0.0626)$ $\mathrm{H} / \mathrm{M}$ ratios in TDT patients.

Correlation of ${ }^{123}$ I-MIBG myocardial uptake and disease duration. ${ }^{123}$ I-MIBG myocardial uptake was significantly correlated with disease duration in early $\left(\mathrm{r}^{2}=0.1894, \mathrm{P}=0.0028\right)$ and delayed images $\left(\mathrm{r}^{2}=0.1795, \mathrm{P}=0.0037\right)$ in $\mathrm{PD}$ patients. The same correlation was found with this parameter when considering the ART population in early $\left(\mathrm{r}^{2}=0.2651, \mathrm{P}=0.0051\right)$ and delayed images $\left(\mathrm{r}^{2}=0.2193, \mathrm{P}=0.0120\right)$. No significant relationships were detected with disease duration in the TDT population in either the early $\left(\mathrm{r}^{2}=0.0916 ; \mathrm{P}=0.2375\right)$ or delayed $\left(r^{2}=0.0983 ; \mathrm{P}=0.2204\right)$ image set.

Correlation of ${ }^{123}$ I-MIBG myocardial uptake and age. No significant correlation was found when considering ${ }^{123}$ I-MIBG myocardial uptake and age in $\mathrm{PD}$ patients at examination in early $(r=-0.1506, P=0.2817)$ and delayed images $(r=-0.2430$, $\mathrm{P}=0.0796)$. The same results were obtained in the $\mathrm{CG}$ in the early $(r=-0.2687, P=0.2970)$ and delayed images $(r=-0.2221$, $\mathrm{P}=0.3916$ ). Considering the single subsets of the PD population, no significant correlations were found with aging in early $(r=-0.1905, P=0.2806)$ and delayed $(r=-0.2700, P=0.1225)$ images and with the $\mathrm{H} / \mathrm{M}$ ratio of ART patients and TDT patients in early $(\mathrm{r}=0.0537, \mathrm{P}=0.8272)$ and delayed images ( $\mathrm{r}=-0.04178, \mathrm{P}=0.8652$ ).

\section{Discussion}

One of the key findings of the present study was the reduction of ${ }^{123} \mathrm{I}-\mathrm{MIBG}$ myocardial uptake in PD patients as compared to the $\mathrm{CG}$ patients. This finding was more marked for the delayed compared to the early images (Figs. 1 and 2), in agreement with several previous studies $(4,7,8,16-20)$. The use of ${ }^{123} \mathrm{I}-\mathrm{MIBG}$ has been suggested as a useful marker in the diagnosis of PD (19) and as a sensitive tool in the early detection of silent autonomic dysfunction (18). Being an analogue of noradrenaline (8), ${ }^{123}$ I-MIBG uptake reflects presynaptic sympathetic integrity, while a reduced myocardial uptake of this tracer is consistent with cardiac sympathetic dysfunction or denervation (21). Nevertheless, Matsui et al demonstrated that there were no significant correlations between orthostatic hypotension or constipation and cardiac ${ }^{123}$ I-MIBG uptake, indicating that not all components of the autonomic nervous system are uniformly affected in PD (19).

In the population of this study, a strong contribution to the lower H/M count-ratio in PD patients as compared with the CG was due to the TDT patients. When the single ART population was analyzed, only the early, but not the delayed, $\mathrm{H} / \mathrm{M}$ ratio was lower than the $\mathrm{CG}$.

In the comparison between TDT and ART, the TDT group showed markedly reduced $\mathrm{H} / \mathrm{M}$ values in the delayed images (Figs. 1 and 2). Moreover, TDT patients showed a reduction of the $\mathrm{H} / \mathrm{M}$ ratio in the delayed images as compared to the early images, while no differences were found in the ART patients. These latter findings are consistent with an increased wash-out of ${ }^{123} \mathrm{I}-\mathrm{MIBG}$ in the myocardium of TDT patients (18), and together with previously mentioned results, indicate a more extensive involvement of the cardiac sympathetic system (due to denervation, a reduced uptake by storage vesicles, abnormalities in reuptake function, and increased norepinephrine release or an increased catecholamine turnover) in PD patients with the tremulous phenotype (22).

Previous studies have shown that in the early stage of PD the dopaminergic system is more severely impaired in ART compared to the other subgroups of patients, in particular when compared to TDT $(11,12)$. To the best of our knowledge, only a few studies have evaluated the ${ }^{123}$ I-MIBG myocardial uptake in different Parkinson's phenotypes $(7,8,20)$. In their study, Spiegel et al found that myocardial ${ }^{123}$ I-MIBG uptake was significantly less impaired in TDT patients than in ART patients at the same stage of the disease (8). Similar results were demonstrated by Saiki et al when comparing a tremor phenotype population of 
PD with a phenotype characterized by postural instability gait difficulty, which could correspond to the ART phenotype (20). A significant correlation was found when comparing the early and delayed $\mathrm{H} / \mathrm{M}$ ratio with the severity of hypokinesia and rigidity, a finding showing that the $\mathrm{H} / \mathrm{M}$ ratio was negatively associated with the severity of these clinical features (8), whereas no correlations have been found with the severity of the resting tremor (8). By contrast, Suzuki et al did not find such a correlation in their population where ${ }^{123}$ I-MIBG myocardial uptake was correlated only with the severity of bradykinesia but not with tremor or rigidity (7). In our study, ${ }^{123}$ I-MIBG myocardial uptake was significantly lower in TDT patients when compared to ART patients $(\mathrm{P}=0.0167)$, findings that are in contrast with those of other studies $(7,8,20)$. These discrepancies may be due to the different disease duration in these two subgroups. Even if differences in disease duration between TDT and ART patients did not reach statistical significance, TDT patients showed a longer disease duration than ART, which potentially affected our results. However, while the $\mathrm{H} / \mathrm{M}$ ratio was significantly associated with disease duration in ART patients, no such correlation was found in TDT patients, supporting the hypothesis that a longer illness duration does not correlate with a worsening of the sympathetic system in this last group. Moreover, no difference was detected beween ART and TDT patients in H\&Y stage, with TDT patients scoring better than ART patients in UPDRS III, reflecting a lower disability level in these patients (9).

No significant correlations were found in either early or delayed images with H\&Y stage and UPDRS motor score in PD patients. Additionally, no statistically significant relationships were evident when comparing the single populations of ART or TDT patients with these aspects of the disease. The lack of a clear relationship of ${ }^{123}$ I-MIBG myocardial uptake with disease severity detectable by means of UPDRS and H\&Y stage has been previously described $(8,19)$. In their study performed on a pool of 143 patients, Suzuki et al hypothesized that $61.3 \%$ of the variation in the observed early $\mathrm{H} / \mathrm{M}$ ratio and $68.2 \%$ of that in the delayed $\mathrm{H} / \mathrm{M}$ ratio could be explained by their linear relationship with disease duration, gender, H\&Y stages and the clinical features of their population (7). Nevertheless, their data indicated no such correlation with clinical disease severity of PD (H\&Y stage) and ${ }^{123}$ I-MIBG myocardial uptake (7). A comparison of both early and delayed $\mathrm{H} / \mathrm{M}$ ratios of $\mathrm{PD}$ patients with disease duration yielded a lower $\mathrm{H} / \mathrm{M}$ ratio in patients with a longer disease duration. In our population, the mean disease duration was $50.7 \pm 60.6$ months, which was lower as compared to that reported in previously published studies in which the same relationship was found and the disease duration was $86.4 \pm 73.2$ months (18). This finding suggests that cardiac sympathetic dysfunction occurs relatively early in PD and the longer the disease duration, the lower the myocardial ${ }^{123}$ I-MIBG uptake and the more sympathetic degeneration occurred. In their study, Kashihara et al found that ${ }^{123}$ I-MIBG myocardial uptake was lower in patients with a long history of PD and the duration of the illness was inversely correlated with the $\mathrm{H} / \mathrm{M}$ ratio (18). Our data suggest that myocardial sympathetic impairment detectable in PD remains with a worsening in the disease course over time (23). However, such a correlation is controversial (24). It has been shown that disease duration was not significantly correlated with either the early or delayed
$\mathrm{H} / \mathrm{M}$ ratio in a pool of 88 patients with $\mathrm{PD}$, while both age at examination and age at onset were negatively correlated (17). Hamada et al suggested that a later onset of the disease rather than the length of disease duration may be associated with a lower ${ }^{123}$ I-MIBG uptake in PD. Our results are slightly different from those of Hamada et al when considering disease duration and the number of subjects examined, and it is possible that a larger standard deviation in our data may play a role in these discrepancies (17).

Of note, no significant differences were detected in cardiac ${ }^{123} \mathrm{I}-\mathrm{MIBG}$ uptake in terms of aging in either CG or PD patients. This observation is in agreement with a previous study reporting a reduced ${ }^{123} \mathrm{I}-\mathrm{MIBG}$ uptake in the inferior heart regions in older patients, but no significant decrease in the whole myocardium with age (1).

In this study, the planar $\mathrm{H} / \mathrm{M}$ values for quantification in the CG (early $1.97 \pm 0.28$, and delayed $1.88 \pm 0.27$ ) were similar to the values reported by Somsen et al and Merlet et al in healthy volunteers $(1,25)$. Morozumi et al reported higher values of early and delayed $\mathrm{H} / \mathrm{M}$ ratios; $2.80 \pm 0.25$ and $3.02 \pm 0.47$, respectively (26). Differences in these measurements have already been explained with the ROI size for the upper mediastinum and the matrix size used (1). In particular, the small ROI used by Morozumi et al for non-specific mediastinal uptake is less affected by non-specific lung uptake leading to a lower mediastinal count density and a larger variability with a larger SD for late H/M (26). Notably, in the CG, differences in the early and delayed $\mathrm{H} / \mathrm{M}$ ratios were statistically significant (Fig. 1). This finding is in agreement with other studies that reported a decrease in $\mathrm{H} / \mathrm{M}$ ratio from early and delayed images in normal subjects $(17,27,28)$. Nevertheless, several studies reported a stability or an increase in the $\mathrm{H} / \mathrm{M}$ ratio over time (1,20,25). A high interindividual and within-subject variability in ${ }^{123}$ I-MIBG uptake has been shown and may explain the differences with the previously mentioned studies (1).

In conclusion, the results of the present study have shown that the cardiac sympathetic system is more severely impaired in TDT than in ART patients. Thus, in contrast to the other movement symptoms of PD, tremor is caused by separate mechanisms and is anatomically different from rigidity and bradykinesia $(29,30)$. Therefore, resting tremor is comparatively less affected by dopamine than other motor symptoms, and the evidence of lesser efficacy of dopaminergic therapy on PD tremor than on other main symptoms emphasizes the contribution of non-dopaminergic mechanisms (12). The scintigraphic findings in TDT are possibly associated with a greater autonomic impairment in these patients with ${ }^{123}$ I-MIBG molecular imaging being capable of improving therapeutic planning. However, more studies on this subject are required.

\section{References}

1. Somsen GA, Verberne HJ, Fleury E and Righetti A: Normal values and within-subject variability of cardiac I-123 MIBG scintigraphy in healthy individuals: Implications for clinical studies. J Nucl Cardiol 11: 126-133, 2004.

2. Braak H, Ghebremedhin E, Rüb U, Bratzke H and Del Tredici K: Stages in the development of Parkinson's disease-related pathology. Cell Tissue Res 318: 121-134, 2004.

3. Kline R C, Swanson DP, Wieland DM, et al: Myocardial imaging in man with I-123 meta-iodobenzylguanidine. J Nucl Med 22: 129-132, 1981. 
4. Spiegel J, Möllers MO, Jost WH, et al: FP-CIT and MIBG scintigraphy in early Parkinson's disease. Mov Disord 20: 552-561, 2005.

5. Ahlskog JE: Diagnosis and differential diagnosis of Parkinson's disease and parkinsonism. Parkinsonism Relat Disord 7: 63-70, 2000.

6. Quinn N: Parkinsonism-recognition and differential diagnosis. Br Med J 310: 447-452, 1995.

7. Suzuki M, Urashima M, Oka H, Hashimoto M and Taira K Cardiac sympathetic denervation in bradykinesia-dominant Parkinson's disease. Neuroreport 18: 1867-1870, 2007.

8. Spiegel J, Hellwig D, Farmakis G, et al: Myocardial sympathetic degeneration correlates with clinical phenotype of Parkinson's disease. Mov Disord 22: 1004-1008, 2007.

9. Hughes A, Daniel SE, Kilford L and Lees AJ: Accuracy of clinical diagnosis of idiopatic Parkinson's disease: a clinico-pathological study of 100 cases. J Neurol Neurosurg Psychiatry 55: 181-184, 1992.

10. Fahn S, Elton RL and members of the UPDRS Development Committee: Unified Parkinson's disease rating scale. Macmillan Healthcare Information, Florhan Park HJ, 1987.

11. Spiegel J, Hellwing D, Samnick S, et al: Striatal FP-CIT uptake differs in the subtypes of early Parkinson's disease. J Neural Transm 114: 331-335, 2007.

12. Schillaci O, Chiaravalloti A, Pierantozzi M, et al: Different patterns of nigrostriatal degeneration in tremor type versus the akinetic-rigid and mixed types of Parkinson's disease at the early stages: molecular imaging with 123I-FP-CIT SPECT. Int J Mol Med 28: 881-886, 2011

13. Lewis SJG, Foltynie T, Blackwell AD, Robbins TW, Owen AM and Barker RA: Heterogeneity of Parkinson's disease in the early clinical stages using a data driven approach. J Neurol Neurosurg Psychiatry 76: 343-348, 1995.

14. Kang GA, Bronstein JM, Masterman DL, et al: Clinical characteristics in early Parkinson's disease in a central California population-based study. Mov Disord 20: 1133-1142, 1995.

15. Solanki KK, Bomanji J, Moyes J, Mather SJ, Trainer PJ and Britton KE: A pharmacological guide to medicines which interfere with the biodistribution of radiolabelled meta-iodobenzylguanidine (MIBG). Nucl Med Commun 14: 513-521, 1992.

16. Courbon F, Brefel-Courbon C, Thalamas C, et al: Cardiac MIBG scintigraphy is a sensitive tool for detecting cardiac sympathetic denervation in Parkinson's disease. Mov Disord 18: 890-897, 2003.

17. Hamada K, Hirayama M, Watanabe $\mathrm{H}$, et al: Onset age and severity of motor impairment are associated with reduction of myocardial 123I-MIBG uptake in Parkinson's disease. J Neurol Neurosurg Psychiatry 74: 423-426, 2003.
18. Kashihara K, Ohno M, Kawada S and Okumura Y: Reduced cardiac uptake and enhanced washout of 123I-MIBG in pure autonomic failure occurs conjointly with Parkinson's disease and dementia with lewy bodies. J Nucl Med 47: 1099-1101, 2006.

19. Matsui H, Nishinaka K, Oda M, Komatsu K, Kubori T and Udaka F: Does cardiac metaiodobenzylguanidine (MIBG) uptake in Parkinson's disease correlate with major autonomic symptoms? Parkinsonism Relat Disord 12: 284-288, 2006.

20. Saiki S, Hirose G, Sakai K, et al: Cardiac 123I-MIBG scintigraphy can assess the disease severity and phenotype of PD. J Neurol Sci 220: 105-111, 2004

21. Hakusui S, Yasuda T, Yanagi T, et al: A radiological analysis of heart sympathetic functions with meta-[123I]iodobenzylguanidine in neurological patients with autonomic failure. J Auton Nerv Syst 49: 81-84, 1994.

22. Pace L, Betocchi S, Losi MA, et al: Sympathetic nervous function in patients with hypertrophic cardiomyopathy assessed by [123I]-MIBG: relationship with left ventricular perfusion and function. Q J Nucl Med Mol Imaging 48: 20-25, 2004.

23. Sawada H, Hoeda T, Yamamoto K, et al: Diagnostc accuracy of cardiac metaiodobenzylguanidine scintigraphy in Parkinson disease. Eur J Neurol 16: 174-182, 2009.

24. Blin J, Dubois B, Bonnet AM, Vidailhet M, Brandabur M and Agid Y: Does ageing aggravate parkinsonian disability? J Neurol Neurosurg Psychiatry 54: 780-782, 1991.

25. Merlet P, Benvenuti C, Moyse D, et al: Prognostic value of MIBG imaging in idiopathic dilated cardiomyopathy. J Nucl Med 40: 917-923, 1999.

26. Morozumi T, Kusuoka H, Fukuchi K, et al: Myocardial iodine123-metaiodobenzylguanidine images and autonomic nerve activity in normal subjects. J Nucl Med 38: 49-52, 1997.

27. Kobayashi S, Tateno M, Morii H, Utsumi K and Saito T: Decreased cardiac MIBG uptake, its correlation with clinical symptoms in dementia with Lewy bodies. Psychiatry Res 174: 76-80, 2009.

28. Yoshita M, Taki J, Yokoyama K, et al: Value of 123I-MIBG radioactivity in the differential diagnosis of DLB from AD. Neurology 66: 1850-1854, 2006.

29. Carr J: Tremor in Parkinson's disease. Parkinsonism Relat Disord 8: 223-234, 2002.

30. Rivlin-Etzion M, Marmor O, Heimer G, Raz A, Nini A and Bergman H: Basal ganglia oscillations and pathophysiology of movement disorders. Curr Opin Neurobiol 16: 629-637, 2006. 